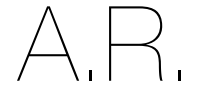

ARTIGO DE REVISÃO

${ }^{1}$ Faculdade de Ciências

da Nutrição e Alimentação da Universidade do Porto, Faculdade de Ciências da Universidade do Porto, Rua do Campo Alegre, n. ${ }^{\circ} 823$,

4150-180 Porto, Portugal

2 EPIUnit, Instituto

de Saúde Pública da

Universidade do Porto,

Rua das Taipas, n. ${ }^{\circ} 135$,

4050-091 Porto, Portugal

${ }^{3}$ LAQV/REQUIMTE,

Laboratório de

Bromatologia e Hidrologia,

Departamento de Ciências

Químicas da Faculdade de

Farmácia da Universidade

do Porto,

Rua Jorge de Viterbo

Ferreira, n. ${ }^{\circ} 228$

4050-313 Porto, Portugal

${ }^{4}$ CIAFEL, Centro de

Investigação em Atividade

Física, Saúde e Lazer da

Faculdade de Desporto da

Universidade do Porto,

Rua Dr. Plácido da Costa,

ก. ${ }^{\circ} 91$

4200-450 Porto, Portugal

${ }^{5}$ CITAB, Centro de Investigação e Tecnologias Agroambientais

Biológicas da Universidade de Trás-os-Montes e Alto

Douro,

Quinta de Prados, Edifício

Reitoria Room D2.30,

5000-801 Vila Real,

Portugal

\section{*Endereço para correspondência:}

Carla Gonçalves

Faculdade de Ciências da

Nutrição e Alimentação

da Universidade do Porto

Faculdade de Ciências da

Universidade do Porto,

Rua do Campo Alegre, n. ${ }^{\circ} 823$

4150-180 Porto, Portugal

carlagoncalves.pt@gmail.com

Histórico do artigo:

Recebido a 16 de fevereiro de 202 Aceite a 10 de maio de 2021

\title{
INTERVENÇÕES PARA A REDUÇÃO DO SAL EM CANTINAS
}

\author{
INTERVENTIONS TO REDUCE SALT IN CANTEENS
}

Inês Mota'; Patrícia Padrão, 2; Tânia Silva-Santos'; Olívia Pinho', 3; Carla Gonçalves ${ }^{1,4,5^{*}}$

RESUMO

O consumo excessivo de sal é um fator de risco para o desenvolvimento de doenças crónicas não transmissíveis como a hipertensão arterial, doenças cardiovasculares e alguns tipos de cancro. Atualmente é frequente a realização de pelo menos uma refeição diária fora de casa, em cantinas ou restaurantes, não havendo controlo da confeção destas refeições por parte do consumidor. O objetivo desta revisão sistemática foi analisar estudos de intervenção para a redução de sal em cantinas públicas ou privadas, nomeadamente avaliar quais as metodologias utilizadas. Foi realizada uma pesquisa nas plataformas PubMed e Web Of Knowledge (entre 23 e 28, setembro 2020), tendo sido incluídos sete artigos. As estratégias adotadas agrupam-se em três áreas principais: ementas e ambiente alimentar, consumidores e cozinheiros/manipuladores de alimentos. As intervenções que resultaram numa redução do consumo de sal foram as alterações das ementas, a formação dos cozinheiros (por cozinheiro ou nutricionista), a substituição do sal de adição por especiarias, a realização de sessões de educação alimentar para os consumidores e a redução do número de refeições pré-embaladas com teor de sal acima de 200 mg/porção fornecidas pelas cantinas. Futuramente será importante realizar estudos com maior uniformização dos procedimentos de avaliação do impacto das estratégias, com um maior período de intervenção e com um grupo controlo, de modo a obter resultados que efetivamente permitam a elaboração de recomendações nesta área.

\section{PALAVRAS-CHAVE}

Cantinas, Intervenção, Redução de sal

ABSTRACT

The excessive consumption of salt is a risk factor for the development of non-transmissible chronic diseases like hypertension, cardiovascular diseases, and cancer. Nowadays it is frequent to have at least one daily meal out of the house, in canteens or restaurants, where there isn't any control, by the customer, with the meals produced. This paper is a systematic review of the existing intervention studies on salt reduction in public or private canteens and evaluate the used methodologies. The search was made on the platforms PubMed and Web of Knowledge $(n=72)$, where, after the proper screening, seven studies fitted the criteria. The methodologies used in the studies that had significant results when it came to reducing salt consumption were changes in the menu, training cookers (with the help of a cooker or nutritionist), substituting added salt for spices, food education sessions for the consumers, and reducing the number of pre-packaged meals with more than $200 \mathrm{mg} /$ portion of salt supplied in canteens. In the future, it would be important to perform studies with greater uniformity of the evaluation procedures of the strategies' impact, with a larger intervention period and a control group, to obtain accurate results, to elaborate recommendations in this area.

\section{KEYWORDS}

Canteens, Intervention, Salt reduction

\section{INTRODUÇÃo}

O consumo excessivo de sal é um problema de saúde pública reconhecido a nível mundial, sendo um fator de risco para vários problemas de saúde como o cancro no estômago, a hipertensão e as doenças cardiovasculares (1-3). Aliás, reconhece-se que $47 \%$ dos casos de doenças cardiovasculares em todo o Mundo sejam causados por hipertensão, associada frequentemente ao consumo excessivo de sal (4). De modo a controlar este grave problema de saúde pública, a Organização Mundial da Saúde (OMS) recomenda que a quantidade de sal a ser consumida diariamente, incluindo tanto o sal presente naturalmente nos alimentos como o de adição, não deve ultrapassar $5 \mathrm{~g} /$ dia (2000 mg/dia de sódio) nos adultos e $3 \mathrm{~g} / \mathrm{dia}$ (1200 mg/dia de sódio) nas crianças com idades entre 2 e 15 anos $(5,6)$.

A alimentação saudável é importante para o correto desenvolvimento e crescimento do organismo, assim como para o desenvolvimento do paladar por alimentos saudáveis, devendo ser iniciada desde idade precoce, de modo a evitar o aparecimento de hipertensão e doenças cardiovasculares, que poderão acompanhar os indivíduos durante toda a sua vida se não forem prevenidas (7-9). Durante grande parte da sua vida, a população faz refeições 
fora de casa, recorrendo principalmente a cantinas e restaurantes, não tendo controlo sobre o sal usado na confeção $(10,11)$. Lin e Frazão verificaram que as refeições confecionadas fora de casa tendem a ter maior teor de gordura e sódio e menor quantidade de cálcio e fibras (10). Num estudo realizado por Barbosa et al. foi avaliado o teor de sódio presente em sopas e pratos principais servidos em sete cantinas universitárias portuguesas e concluíram que o teor de sódio dos componentes alimentares estava acima do recomendado: os pratos principais continham uma média de 671,4 × 374,5 mg de sódio/porção, sendo que os pratos de peixe e vegetarianos tinham maior teor de sódio comparativamente aos pratos de carne, e as sopas continham em média 398,0 \pm 153,8 mg de sódio/porção (12). Aliás, Gonçalves et al. verificaram, num estudo anterior, que o principal responsável pela quantidade de sódio presente nas sopas era o uso de sal de adição (acima de 90\% do sódio total), apresentando valores elevados, principalmente em infantários e lares de idosos (13).

Este trabalho pretende realizar uma revisão sistemática sobre estudos de intervenção para a redução de sal em cantinas públicas ou privadas, nomeadamente analisar quais as metodologias utilizadas.

\section{METODOLOGIA}

Os métodos do presente trabalho de revisão foram escritos tendo em consideração as recomendações internacionais (14).

A pesquisa destes artigos foi feita nas plataformas PubMed e Web of Knowledge, entre 23 e 28 de setembro de 2020. Esta foi feita por uma das autoras, tendo em consideração termos e palavras-chave relacionadas com cantinas ou empresas de restauração e intervenção para diminuição do uso de sal. Os termos pesquisados em ambas as plataformas foram (("salt" OR "sodium" OR "sodium chloride") AND ("canteen" OR "cafeteria" OR "catering") AND ("randomized controlled trial" OR "trial" OR "intervention")).

Foram considerados os seguintes critérios de elegibilidade: 1) estudos realizados em cantinas públicas ou privadas ou cafetarias; 2) estudos de intervenção; 3) estudos em que é avaliado o teor de sal antes e após a intervenção; 4) estudos escritos em português ou inglês.

Foi realizada a seguinte extração de dados, com consenso entre duas autoras: o apelido do primeiro autor, o ano de publicação, o país, a população em estudo, o local onde foram realizados os estudos, o período de intervenção, a intervenção, o método de avaliação e os resultados.

\section{RESULTADOS}

A pesquisa nas plataformas permitiu obter setenta resultados. Adicionalmente, foram acrescentados dois estudos incluídos nas referências bibliográficas dos artigos encontrados. Após a remoção dos duplicados $(n=21)$, recorreu-se a uma primeira fase de leitura dos títulos e devidos resumos, excluindo dez artigos, por serem artigos focados em animais, plantas e produtos alimentares. Após esta etapa de triagem, fez-se a leitura na íntegra dos trinta e nove artigos selecionados e concluiu-se que apenas sete estudos se adequavam aos critérios de elegibilidade. Foram excluídos trinta e dois artigos, pelos motivos: estudos observacionais $(n=10)$, estudos onde não houve intervenção para redução de sal $(n=14)$, estudos que são protocolos $(n=2)$, estudos não realizados em cantinas $(n=4)$, estudos que não visaram a redução de sal $(n=1)$ e estudos que se focaram em comentar outros estudos realizados $(n=1)$.

A Tabela 1 contém o resumo das características dos sete artigos incluídos ordenados por ano de publicação. Os estudos foram realizados em diferentes países, sendo que três decorreram no continente americano, quatro na Europa, não tido sido incluído nenhum estudo realizado em Portugal. O período de intervenção dos estudos incluídos variou entre duas semanas e dezoito meses. Todos os estudos envolveram consumidores de ambos os sexos pertencentes a diferentes faixas etárias.

\section{Estratégias para Redução do Sal em Cantinas}

Nos estudos analisados, foram utilizadas diferentes estratégias para atingir o objetivo de redução do sal, sendo possível considerar o seu agrupamento em três domínios.

\section{Alteração de ementas e do ambiente alimentar}

No estudo de Geaney et al., as ementas foram alteradas de modo a diminuir o consumo de sal, havendo a substituição dos produtos processados e ricos em sal por produtos frescos e com pouco teor de sal, nesta intervenção os participantes do grupo de intervenção mostraram, em média, menor consumo de sal em comparação com o grupo controlo, 5,6 g/dia de sal vs. 6,7 g/dia de sal, respetivamente, $p<0,046$ (15). No estudo de Janssen et al., utilizaram produtos processados com redução de sal pela indústria, totalizando uma redução de $29 \%$ a $61 \%$ do sal dos alimentos disponíveis na cantina. Avaliaram a quantidade de sódio excretada na urina de 24 horas dos consumidores do grupo de intervenção e esta foi significativamente inferior à do grupo de controlo (119 mEq/dia vs. $165 \mathrm{mEq} /$ dia, $\mathrm{p}<0,05)$. (16). No estudo de Cohen et al., houve a implementação de uma nova ementa, elaborada por um cozinheiro profissional, contendo menor teor de açúcar, gorduras e sal. Após a intervenção verificou-se nas escolas de intervenção, o sódio das refeições era menor do que nas escolas do grupo controlo (927mg vs. $1211 \mathrm{mg}, \mathrm{p}<0,0001)$ (17). Estas estratégias provaram ser eficazes, visto que nos três estudos, os resultados obtidos após a implementação da intervenção para a redução de sal foram significativos. O estudo de Brooks et al. foi o único que teve como objeto de estudo as máquinas de venda automática, cafetarias e quiosques, pretendendo reduzir a quantidade de produtos alimentares embalados com teores de sal superior a 200 mg por porção nestes locais, o que mostrou ter sucesso, havendo uma diminuição da quantidade destas refeições nos locais de venda (18). No estudo de Reynoso-Marreros et al., o sal adicionado às refeições foi padronizado, no momento da confeção culinária, com uma redução de $20 \%$ e foram removidos os saleiros de mesa. Verificou-se que o sal de adição na confeção de refeições diminuiu, embora os resultados não tenham sido significativos, observou-se uma diminuição significativa da pressão arterial dos consumidores, sem haver variação do desperdício alimentar e dos testes hedónicos (19).

\section{Intervenções nos consumidores}

A intervenção realizada por Geaney et al., nos consumidores foi a apresentação de informação sobre a redução do sal e alimentação saudável, que demonstrou ser eficaz na diminuição do consumo de sal, os participantes do grupo de intervenção avaliados pelo questionário recordatório das $24 \mathrm{~h}$ anteriores apresentaram, em média, uma diminuição significativa do consumo de sal. Embora esta estratégia tenha sido combinada com a alteração das ementas e remoção dos saleiros de mesa (15). Na intervenção descrita por Brooks et al., forneceram aos participantes materiais educacionais para identificar opções saudáveis com baixo teor de sódio e aumentar a conscientização do consumidor sobre os efeitos na saúde, no entanto os autores não avaliaram o impacto da intervenção na redução do consumo de sal pelos participantes (18). Na intervenção descrita por Beer-Borst et al. realizaram workshops, enviaram emails e links para jogos ou filmes e trabalhos práticos sobre a importância da redução 


\begin{tabular}{|c|c|c|c|c|c|c|c|c|}
\hline $\begin{array}{l}\text { AUTOR(ES) } \\
\text { (ANO) }\end{array}$ & PAís & $\begin{array}{c}\text { TIPO } \\
\text { DE ESTUDO }\end{array}$ & $\begin{array}{l}\text { OBJETO } \\
\text { DE ESTUDO }\end{array}$ & $\begin{array}{c}\text { LOCAL } \\
\text { DE ESTUDO }\end{array}$ & $\begin{array}{l}\text { PERÍODO DE } \\
\text { INTERVENÇÃO }\end{array}$ & INTERVENÇÃO & $\begin{array}{l}\text { MÉTODO DE } \\
\text { AVALIAÇÃO }\end{array}$ & RESULTADOS \\
\hline $\begin{array}{l}\text { Geaney } \\
\text { et al. } \\
\text { (2011) } \\
\text { (15) }\end{array}$ & Irlanda & $\begin{array}{l}\text { Estudo piloto, } \\
\text { transversal de } \\
\text { comparação }\end{array}$ & $\begin{array}{l}100 \text { trabalhadores de } 2 \\
\text { hospitais, com idades } \\
\text { compreendidas entre } \\
\text { os } 18 \text { e } 64 \text { anos ( } 50 \text { no } \\
\text { grupo de intervenção e } \\
50 \text { no grupo de controlo) } \\
\text { que consomem pelo } \\
\text { menos uma refeição } \\
\text { principal na cantina do } \\
\text { hospital por dia }\end{array}$ & $\begin{array}{l}2 \text { cantinas de } \\
\text { hospitais do setor } \\
\text { público }\end{array}$ & não definido & $\begin{array}{l}\text { Alteração das ementas, } \\
\text { substituindo produtos } \\
\text { processados e ricos em } \\
\text { sal por produtos frescos } \\
\text { e com pouco teor de } \\
\text { sal. Introdução de ervas } \\
\text { aromáticas e especiarias. } \\
\text { Remoção do sal em } \\
\text { todos os processos de } \\
\text { confeção e os saleiros } \\
\text { disponíveis na mesa } \\
\text { foram substituídos } \\
\text { por sacos individuais } \\
\text { de sal disponíveis no } \\
\text { serviço. Apresentação } \\
\text { de informaçãa nutricional } \\
\text { sobre redução de sal e } \\
\text { alimentaçáo saudável. }\end{array}$ & $\begin{array}{l}\text { Questionário das } \\
24 \mathrm{~h} \text { anteriores. }\end{array}$ & $\begin{array}{l}\text { Os participantes do } \\
\text { grupo de intervenção } \\
\text { mostraram, em média, } \\
\text { menor consumo de sal } \\
\text { em comparação com o } \\
\text { grupo controlo, } 5,6 \mathrm{~g} / \\
\text { dia de sal vs. } 6,7 \mathrm{~g} / \text { dia } \\
\text { de sal, respetivamente, } \\
\mathrm{p}<0,046 \text {. }\end{array}$ \\
\hline
\end{tabular}

\begin{tabular}{|c|c|c|c|c|c|c|c|c|}
\hline $\begin{array}{l}\text { Cohen } \\
\text { et al. } \\
(2012) \\
(17)\end{array}$ & $\begin{array}{l}\text { Estados } \\
\text { Unidos da } \\
\text { América }\end{array}$ & $\begin{array}{l}\text { Estudo piloto, } \\
\text { transversal de } \\
\text { comparação }\end{array}$ & $\begin{array}{l}3049 \text { estudantes (1609 } \\
\text { estudantes nas escolas } \\
\text { do grupo de intervenção } \\
\text { e } 1440 \text { estudantes } \\
\text { nas escolas do grupo } \\
\text { controlo) }\end{array}$ & $\begin{array}{l}19 \text { cantinas de } \\
\text { escolas públicas } \\
\text { de ensino médio }\end{array}$ & 2 anos & $\begin{array}{l}\text { Formação dos cozinheiros } \\
\text { e implementação } \\
\text { de novas ementas } \\
\text { elaboradas por um } \\
\text { chef profissional para a } \\
\text { preparação de refeições } \\
\text { com menor teor de } \\
\text { açúcar, gorduras trans, } \\
\text { gorduras saturadas e sal. }\end{array}$ & $\begin{array}{l}\text { Análise da } \\
\text { composição } \\
\text { das refeições } \\
\text { disponibilizadas } \\
\text { através de } \\
\text { tabelas de } \\
\text { composição } \\
\text { nutricional e } \\
\text { rótulos dos } \\
\text { alimentos. } \\
\text { Desperdício } \\
\text { alimentar. }\end{array}$ & $\begin{array}{l}\text { Nas escolas do grupo de } \\
\text { intervenção verificou-se } \\
\text { que o teor de sódio das } \\
\text { refeições, em média, } \\
\text { era menor do que } \\
\text { nas escolas do grupo } \\
\text { controlo ( } 927 \mathrm{mg} \text { vs. } 1211 \\
\mathrm{mg}, \mathrm{p}<0,0001) \text {. } \\
\text { Não existiram diferenças } \\
\text { significativas do } \\
\text { desperdício alimentar } \\
\text { entre os dois grupos } \\
(61.6 \% \text { vs. } 57.3 ; \\
\mathrm{p}=0,630) \text {. }\end{array}$ \\
\hline $\begin{array}{l}\text { Dorresteijn } \\
\text { et al. (2012) } \\
\text { (21) }\end{array}$ & Holanda & $\begin{array}{l}\text { Estudo de } \\
\text { intervenção, } \\
\text { simples, cego }\end{array}$ & $\begin{array}{l}\text { Trabalhadores e } \\
\text { visitantes do hospital }\end{array}$ & $\begin{array}{l}\text { Cantina do } \\
\text { centro Hospitalar } \\
\text { Universitário }\end{array}$ & 2 semanas & $\begin{array}{l}\text { Redução do uso de sal } \\
\text { na confeção da sopa } \\
\text { cremosa, de } 2,4 \mathrm{~g} \text { por } \\
200 \mathrm{ml} \text { de sopa para } 1,7 \mathrm{~g} \text {, } \\
\text { aproximadamente } 30 \% \text {. } \\
\text { Uso de cartazes a sinalizar } \\
\text { que "a sopa cremosa } \\
\text { contém menos } 30 \% \text { de } \\
\text { sal e contribui para uma } \\
\text { pressão arterial saudável". }\end{array}$ & $\begin{array}{l}\text { A venda de sopa } \\
\text { cremosa com } \\
\text { baixo teor de sal } \\
\text { vs sopa com teor } \\
\text { normal de sal. }\end{array}$ & $\begin{array}{l}\text { Não se verificou } \\
\text { alteração no } \\
\text { comportamento de } \\
\text { compra do consumidor } \\
\text { nos períodos baseline, } \\
\text { intervenção e após o } \\
\text { período de intervenção. }\end{array}$ \\
\hline $\begin{array}{l}\text { Janssen } \\
\text { el al. } \\
(2015) \\
(16)\end{array}$ & Holanda & $\begin{array}{l}\text { Estudo de } \\
\text { intervenção } \\
\text { randomizado, } \\
\text { simples, cego }\end{array}$ & $\begin{array}{l}74 \text { alunos e funcionários } \\
\text { de uma universidade } \\
\text { ( } 36 \text { indivíduos do grupo } \\
\text { de intervenção e } 38 \text { do } \\
\text { grupo de controlo) }\end{array}$ & $\begin{array}{l}\text { Cantina } \\
\text { experimental }\end{array}$ & $\begin{array}{l}3 \text { semanas } \\
\text { (12 dias) }\end{array}$ & $\begin{array}{l}\text { Cantina experimental } \\
\text { com alimentos que } \\
\text { tiveram uma redução de } \\
29 \text { a } 61 \% \text { de sódio pelos } \\
\text { produtores industriais. } \\
\text { Acrescentaram potássio } \\
\text { e/ou outros aditivos para } \\
\text { aumentar a salinidade. }\end{array}$ & $\begin{array}{l}\text { Análise da } \\
\text { composição } \\
\text { das refeições. } \\
\text { Excreção de sódio } \\
\text { pela urina 24h. }\end{array}$ & $\begin{array}{l}\text { O consumo de } \\
\text { sódio estimado pela } \\
\text { composição das } \\
\text { refeições diminuiu } \\
\text { significativamente no } \\
\text { grupo de intervenção } \\
\text { (1363 mg/dia vs. } 2493 \\
\text { mg/dia). } \\
\text { A quantidade de sódio } \\
\text { excretada pela urina } \\
\text { de } 24 \text { h no grupo } \\
\text { de intervenção foi } \\
\text { significativamente } \\
\text { inferior à do grupo de } \\
\text { controlo (119 mEq/ } \\
\text { dia vs. } 165 \mathrm{mEq} / \mathrm{dia}, \\
\text { respetivamente<0,05). }\end{array}$ \\
\hline $\begin{array}{l}\text { Brooks } \\
\text { et al. } \\
(2017) \\
(18)\end{array}$ & $\begin{array}{l}\text { Estados } \\
\text { Unidos da } \\
\text { América }\end{array}$ & $\begin{array}{l}\text { Quasi- } \\
\text { Experimental sem } \\
\text { grupo de controlo }\end{array}$ & $\begin{array}{l}\text { Máquinas de venda } \\
\text { automática, cafetarias e } \\
\text { quiosques }\end{array}$ & $\begin{array}{l}21 \text { Instituições } \\
\text { comunitárias }\end{array}$ & 12 a 18 meses & $\begin{array}{l}\text { Um relatório com os níveis } \\
\text { atuais de teor de sódio. } \\
\text { Estabelecimento de um } \\
\text { plano de ação com metas } \\
\text { para redução de sódio. } \\
\text { Apoio técnico da nutrição } \\
\text { individualizado, através de } \\
\text { reuniões e chamadas. } \\
\text { Fornecimento de } \\
\text { materiais para facilitar } \\
\text { a implementação das } \\
\text { estratégias de redução } \\
\text { de sal. } \\
\text { Materiais educacionais } \\
\text { para identificar opções } \\
\text { saudáveis com baixo } \\
\text { teor de sódio e aumentar } \\
\text { a conscientização do } \\
\text { consumidor sobre } \\
\text { os efeitos na saúde } \\
\text { associados ao excesso } \\
\text { de sódio. }\end{array}$ & $\begin{array}{l}\text { Redução de } \\
\text { alimentos pré- } \\
\text { embalados com } \\
\text { quantidades de } \\
\text { sódio superiores } \\
\text { a } 200 \mathrm{mg} / \text { porção. }\end{array}$ & $\begin{array}{l}\text { A disponibilidade de } \\
\text { produtos alimentares } \\
\text { pré-embalados que } \\
\text { continham mais de } 200 \\
\text { mg de sódio por porção } \\
\text { diminuiu de } 29 \% \text { para } \\
21,5 \% \text { ( } \mathrm{p}=0,003 \text { ) nos } \\
\text { vários locais de acesso. }\end{array}$ \\
\hline
\end{tabular}




\begin{tabular}{|c|c|c|c|c|c|c|c|c|}
\hline $\begin{array}{l}\text { AUTOR(ES) } \\
\text { (ANO) }\end{array}$ & PAís & $\begin{array}{c}\text { TIPO } \\
\text { DE ESTUDO }\end{array}$ & $\begin{array}{l}\text { OBJETO } \\
\text { DE ESTUDO }\end{array}$ & $\begin{array}{c}\text { LOCAL } \\
\text { DE ESTUDO }\end{array}$ & $\begin{array}{l}\text { PERÍODO DE } \\
\text { INTERVENÇÃOO }\end{array}$ & INTERVENÇÃO & $\begin{array}{l}\text { MÉTODO DE } \\
\text { AVALIAÇẪO }\end{array}$ & RESULTADOS \\
\hline $\begin{array}{l}\text { Beer-Borst } \\
\text { et al. } \\
(2019) \\
(20)\end{array}$ & Suíça & $\begin{array}{l}\text { Quasi- } \\
\text { experimental }\end{array}$ & $\begin{array}{l}141 \text { voluntários, } 70 \\
\text { mulheres e } 71 \text { homens, } \\
\text { com idade entre os } \\
15 \text { e os } 65 \text { anos ( } 128 \\
\text { participantes do grupo } \\
\text { de intervenção e } 13 \text { do } \\
\text { grupo de controlo) }\end{array}$ & $\begin{array}{l}8 \text { locais de } \\
\text { trabalho }\end{array}$ & 12 meses & $\begin{array}{l}\text { Combinação de } \\
\text { intervenção educacional } \\
\text { e de uma intervenção no } \\
\text { ambiente. A intervenção } \\
\text { no ambiente baseou- } \\
\text { se num programa de } \\
\text { coaching (apresentações, } \\
\text { workshops) para } \\
\text { formar os cozinheiros } \\
\text { e conscientizar sobre } \\
\text { os riscos para a saúde } \\
\text { do consumo de sal e } \\
\text { implementarem uma } \\
\text { alimentação balanceada e } \\
\text { saborosa com teor de sal } \\
\text { controlado (2,5 g de sal } \\
\text { por porção de almoço). } \\
\text { A intervenção educacional } \\
\text { foi baseada em dotar } \\
\text { os participantes de } \\
\text { conhecimentos sobre } \\
\text { nutrição e aconselhar } \\
\text { como escolher alimentos } \\
\text { em casa e fora de casa } \\
\text { (workshops, emails, links } \\
\text { para jogos ou filmes, } \\
\text { trabalhos práticos). }\end{array}$ & $\begin{array}{l}\text { Excreção urinária } \\
\text { de } 24 \text { horas. }\end{array}$ & $\begin{array}{l}\text { A quantidade média } \\
\text { de sal consumido } \\
\text { diariamente diminuiu } \\
\text { de } 8,7 \mathrm{~g} \text { para } 8,1 \mathrm{~g} \\
\text { (6,9\% em } 12 \text { meses), } \\
\text { não sendo significativo } \\
\text { ( } \mathrm{p}=0,192) \text {, no grupo de } \\
\text { intervenção. } \\
\text { A razão } \mathrm{Na} / \mathrm{K} \text {, no grupo } \\
\text { de intervenção, no } \\
\text { período baseline foi } 1,1 \\
\text { e no fim do estudo foi } \\
1,3 \mathrm{p}=0,007) \text {. A ingestão } \\
\text { de potássio diminuiu de } \\
3,1 \mathrm{~g} / \text { dia para } 2.6 \mathrm{~g} / \mathrm{dia} \\
\text { ( } \mathrm{p}<0,001) \text {. } \\
\text { Não disponibilizaram os } \\
\text { dados do grupo controlo. }\end{array}$ \\
\hline $\begin{array}{l}\text { Reynoso- } \\
\text { Marreros } \\
\text { et al. } \\
(2019) \\
(19)\end{array}$ & Perú & $\begin{array}{l}\text { Quasi- } \\
\text { Experimental }\end{array}$ & $\begin{array}{l}71 \text { trabalhadores ( } 57,8 \% \\
\text { mulheres) com idades } \\
\text { compreendidas entre os } \\
24 \text { e } 60 \text { anos }\end{array}$ & $\begin{array}{l}\text { Uma empresa } \\
\text { privada }\end{array}$ & 4 semanas & $\begin{array}{l}\text { Padronização do sal } \\
\text { adicionado às refeiçães, } \\
\text { no momento da confeção } \\
\text { culinária, com uma } \\
\text { redução de } 20 \% \text {. } \\
\text { Remoção dos saleiros das } \\
\text { mesas. }\end{array}$ & $\begin{array}{l}\text { Pesagem do } \\
\text { sal usado às } \\
\text { refeições. Medição } \\
\text { da pressão } \\
\text { arterial. } \\
\text { Análise do } \\
\text { desperdício } \\
\text { alimentar. } \\
\text { Avaliação do grau } \\
\text { de satisfação } \\
\text { (escalas de } \\
\text { avaliação } \\
\text { hedónica). }\end{array}$ & $\begin{array}{l}\text { Em média, o uso de sal } \\
\text { de adição na confeção } \\
\text { de refeições diminuiu de } \\
10,3 \mathrm{~g} \text { para } 8,2 \mathrm{~g} \text { (uma } \\
\text { diferença de } 2,1 \mathrm{~g} \text {, que } \\
\text { corresponde a } 20 \% \text { de } \\
\text { sal reduzido), não sendo } \\
\text { significativo. } \\
\text { Após a intervenção, } \\
\text { houve uma diminuição } \\
\text { de } 3,1 \mathrm{mmH} \text { da pressão } \\
\text { sistólica ( } \mathrm{p}<0,001 \mathrm{e} \\
2,9 \mathrm{~mm} \mathrm{Hg} \text { da pressão } \\
\text { diastólica ( } \mathrm{p}<0,001) \text {. } \\
\text { Não houve variação do } \\
\text { desperdício alimentar ao } \\
\text { longo do estudo. } \\
\text { Não houve alterações } \\
\text { significativas nos testes } \\
\text { hedónicos. }\end{array}$ \\
\hline
\end{tabular}

de sal, consequências deste para a saúde e recomendações sobre alimentação saudável, verificou-se uma tendência de diminuição do consumo de sal no fim da intervenção no grupo de intervenção, uma vez que a quantidade média de sal consumido diariamente diminuiu de 8,7 g para 8,1 g (6,9\% em 12 meses), no entanto, sem significado estatístico $(p=0,192)(20)$. Dorresteijn et al., usaram cartazes a informar que a sopa continha menos 30\% de sal e contribuía para uma pressão arterial saudável, não se verificou alteração no comportamento de compra do consumidor nos períodos baseline, intervenção e após o período de intervenção (21).

\section{Intervenção nos manipuladores de alimentos}

As intervenções nos manipuladores de alimentos, foram combinadas com outras estratégias já descritas. Beer-Borst et al., realizaram formações com recurso a um programa de coaching que incluiu apresentações e workshops para conscientizar os cozinheiros sobre os riscos para a saúde do consumo de sal e implementarem uma alimentação equilibrada e saborosa com teor de sal controlado. A quantidade média de sal ingerido pelos consumidores diariamente não diminuiu de forma significativa (20). Cohen et al. descreveram uma intervenção nas escolas, onde foi dada formação aos cozinheiros e implementaram novas ementas elaboradas por um chef profissional. A intervenção teve resultados significativos, verificou-se que o teor de sódio das refeições das escolas do grupo de intervenção, em média, era menor do que nas escolas do grupo controlo (17).

\section{DISCUSSÃO DOS RESULTADOS}

O presente trabalho de revisão sumariza as estratégias e os resultados de sete estudos relevantes com intervenções para a diminuição do uso e consumo de sal em cantinas públicas ou privadas e cafetarias. No estudo de Dorresteijn et al., a intervenção realizou-se durante duas semanas, sendo que os investigadores consideraram que o curto período de intervenção foi uma limitação do estudo e que as intervenções para a redução de sal em estudos clínicos deverão ser superiores a quatro semanas (21-24). Reynoso-Marreros et al. afirmam no seu estudo que este período ajuda significativamente a melhorar a pressão arterial (19).

Relativamente aos participantes no estudo de Geaney et al., verifica-se que estes não têm outras opções alimentares nem restaurantes por perto para se deslocarem, acabando por realizar as suas refeições nas cantinas dos hospitais (15). O objetivo desta intervenção foi fornecer opções de alimentos nutritivas para os funcionários, com foco particular na redução da ingestão de sal. Os pedidos de compra de produtos com alto teor de sal (misturas de molhos, cubos de caldo) e carnes processadas (bacon, carne enlatada) foram substituídos por opções com baixo teor de sal (peru, frango e peixe). Ervas aromáticas frescas, 
especiarias e alho foram introduzidos para desenvolver um sabor adicional e removeram o sal em todos os processos de confeção. Os participantes do grupo de intervenção avaliados pelo questionário das 24h anteriores apresentaram, em média, uma diminuição significativa do consumo de sal. Os investigadores indicam ser importante, no futuro, a realização de testes de urina de $24 \mathrm{~h}$ durante vários dias, incluindo períodos de férias e/ou folgas, de modo a aferir se os participantes realizam refeições saudáveis fora das cantinas dos hospitais (15).

Com uma maior formação, os cozinheiros e manipuladores de alimentos das cantinas serão capazes de fornecer refeições mais saudáveis e saborosas, podendo melhorar os hábitos alimentares dos consumidores e, consequentemente, a sua saúde (17). Segundo Gonçalves et al., que desenvolveram um estudo envolvendo manipuladores de alimentos de uma empresa de restauração, em Portugal e verificaram que estes se preocupam em fornecer refeições saudáveis, tendo conhecimento que o sal em excesso é prejudicial para a saúde e qual o limite máximo diário de consumo definido pela OMS (25). Uma das limitações apontadas para a adoção da redução do sal nas refeições confecionadas é a satisfação e a opinião do consumidor (26), realçando a importância de intervenções que envolvam o aumento da sensibilização e literacia do consumidor para a redução do sal.

Estes estudos avaliaram o impacto da redução de sal em diferentes domínios, como no consumo, através da excreção urinária de 24h, pela análise da composição das refeições servidas através de tabelas de composição nutricional e rótulos dos alimentos e do questionário recordatório das 24 h anteriores $(15,16,20)$, na oferta, através da análise das refeições/ementas e análise dos alimentos pré-embalados $(17,18)$, na venda das refeições $(21)$, no desperdício alimentar $(17,19)$ e na saúde, com a medição da pressão arterial (19). Em relação às avaliações da ingestão de sódio através da urina de 24h, no estudo de Janssen et al. verificou-se que, apesar de consumirem refeições com menor teor de sal na cantina, os participantes do grupo de intervenção não sentiram a necessidade de compensar o sal não consumido. Os autores reduziram entre $29 \%$ e $61 \%$ o sal dos alimentos disponibilizados na cantina através da aquisição de alternativas de alimentos processados com menor teor de sódio (dezanove produtos alimentares reformulados como pão, queijo, sopa, saladas e carne, sendo consideradas aquisição de produtos embalados com baixo sal ou ingredientes processados com menor teor em sal para os confecionar) (16).

Reynoso-Marreros et al. e Dorresteijn et al. indicaram nos seus estudos que é possível reduzir gradualmente o sal a ser adicionado na confeção de refeições e consumido sem afetar a aceitabilidade destas. Dorresteijn et al. estimaram a quantidade de sal adicionado nas duas sopas servidas nos hospitais e reduziram cerca de 30\% na sopa creme, informaram os consumidores da redução através de cartazes e o comportamento de compra do consumidor não se alterou. Reynoso-Marreros et al. procederam a uma redução de $20 \%$ do sal adicionado aos alimentos durante a confeção com base em estudos anteriores que indicaram que essa redução não era percecionada pelos consumidores e não afetava a palatabilidade dos alimentos. Antes da intervenção, os cozinheiros foram treinados para pesar o sal adicionado aos alimentos durante a confeção num período de quatro semanas, posteriormente elaboraram uma lista detalhada das receitas com a quantidade de sal habitualmente adicionada e reduziram $20 \%$ no período de intervenção. $O$ sal foi colocado em saquinhos para garantir um processo adequado sem adicionar quantidade extra $(19,21)$. Relativamente à saúde, ReynosoMarreros et al. demonstraram que houve alterações significativas e positivas das pressões sistólica e diastólica, o que indica que a implementação de intervenções para a redução do uso de sal pode ter um impacto positivo na pressão arterial (19). A quantidade preferida de sal nos alimentos depende da quantidade de sal habitualmente consumido e essa quantidade preferida pode ser reduzida após uma diminuição gradual da ingestão de sal (26), resultando em um aumento na intensidade de sal percebida e na diminuição do gosto por alimentos com teor elevado de sal (27-29).

Futuramente será importante realizar estudos de intervenção que incluam outros locais, como infantários e em Estruturas Residenciais para Pessoas Idosas, e ainda ter em consideração outras doenças crónicas que os participantes possam ter, como diabetes mellitus e hipercolesterolemia (19).

\section{CONCLUSÕES}

Com a realização desta revisão sistemática é possível concluir que, apesar de existirem estudos de intervenção para redução do uso de sal na confeção de refeições em cantinas, estes são insuficientes. Existem diferentes estratégias que podem ser adotadas, que podem ser agrupadas em três áreas principais de intervenção: alteração de ementas/ambiente alimentar, consumidores e manipuladores de alimentos. As intervenções podem ser realizadas numa só área ou em mais do que uma área, devendo ser adaptadas ao contexto específico. As intervenções com alteração das ementas com foco na redução de sal apresentaram resultados positivos na diminuição do consumo de sal e quando as reduções são graduais parece não existir impacto negativo na aceitabilidade dos alimentos pelo consumidor. No futuro, o ideal é voltar a repetir estes estudos, em diferentes ambientes, com um maior período de intervenção e com medidas de adesão objetivas de modo a obter resultados mais fiáveis, sugerindo que metodologias conferem um maior impacto na redução do sal.

\section{FINANCIAMENTO}

Este trabalho foi financiado pela Fundação para a Ciência e Tecnologia através da bolsa POCI-01-0145-FEDER-029269.

\section{REFERÊNCIAS BIBLIOGRÁFICAS}

1. Bibbins-Domingo K, Chertow GM, Coxson PG, Moran A, Lightwood JM, Pletcher MJ, et al. Projected Effect of Dietary Salt Reductions on Future Cardiovascular Disease. New England Journal of Medicine. 2010;362(7):590-9.

2. Cappuccio FP. Cardiovascular and other effects of salt consumption. Kidney Int Suppl (2011). 2013;3(4):312-5.

3. Ezzati M, Lopez AD, Rodgers A, Vander Hoorn S, Murray CJ. Selected major risk factors and global and regional burden of disease. Lancet. 2002;360(9343):1347-60. 4. Afshin A, Sur PJ, Fay KA, Cornaby L, Ferrara G, Salama JS, et al. Health effects of dietary risks in 195 countries, 1990\&\#x2013;2017: a systematic analysis for the Global Burden of Disease Study 2017. The Lancet. 2019;393(10184):1958-72.

5. Nogueira PJB, Ramalhinho DV. Sal e Hipertensão Arterial https://www.sphta.org.pt/ pt/base8_detail/25/105: Sociedade Portuguesa de Hipertensão; 2020 [76:[.

6. WHO. Sodium intake for adults and children https://www.who.int/publications/i/ item/9789241504836: World Health Organization; 2012 [.

7. Chen $X$, Wang Y. Tracking of blood pressure from childhood to adulthood: a systematic review and meta-regression analysis. Circulation. 2008;117(25):3171-80. 8. Rosner B, Cook NR, Daniels S, Falkner B. Childhood blood pressure trends and risk factors for high blood pressure: the NHANES experience 1988-2008. Hypertension. 2013;62(2):247-54.

9. Yang Q, Zhang Z, Kuklina EV, Fang J, Ayala C, Hong Y, et al. Sodium intake and blood pressure among US children and adolescents. Pediatrics. 2012;130(4):611-9. 10. Lin B-H, Frazao E. Nutritional Quality of Foods At and Away From Home. Food Review/ National Food Review. 1997;20(2):234485.

11. Roos E, Sarlio-Lähteenkorva S, Lallukka T. Having lunch at staff canteen is 
associated with recommended food habits. Public health nutrition. 2004;7:53-61.

12. Barbosa MI, Fernandes A, Gonçalves C, Pena MJ, Padrão P, Pinho O, et al. Sodium and Potassium Content of Meals Served in University Canteens. Portuguese Journal of Public Health. 2017;35(3):172-8.

13. Gonçalves C, Silva G, Pinho O, Camelo S, Amaro L, Teixeira V, et al. Sodium Content in Vegetable Soups Prepared Outside the Home: Identifying the Problem2012. 14. Higgins, J. P., Thomas, J., Chandler, J., Cumpston, M., Li, T., Page, M. J., \& Welch, V. A. (Eds.). (2019). Cochrane handbook for systematic reviews of interventions. John Wiley \& Sons.

15. Geaney F, Harrington J, Fitzgerald A, Perry I. The impact of a workplace catering initiative on dietary intakes of salt and other nutrients: a pilot study. Public Health Nutr. 2011;14(8):1345-9.

16. Janssen AM, Kremer S, van Stipriaan WL, Noort MWJ, de Vries JHM, Temme EHM. Reduced-Sodium Lunches Are Well-Accepted by Uninformed Consumers Over a 3-Week Period and Result in Decreased Daily Dietary Sodium Intakes: A Randomized Controlled Trial. Journal of the Academy of Nutrition and Dietetics. 2015;115(10):1614-25.

17. Cohen JFW, Smit LA, Parker E, Austin SB, Frazier AL, Economos CD, et al. LongTerm Impact of a Chef on School Lunch Consumption: Findings from a 2-Year Pilot Study in Boston Middle Schools. Journal of the Academy of Nutrition and Dietetics. 2012;112(6):927-33.

18. Brooks CJ, Barrett J, Daly J, Lee R, Blanding N, McHugh A, et al. A CommunityLevel Sodium Reduction Intervention, Boston, 2013-2015. American Journal of Public Health. 2017;107(12):1951-7.

19. Reynoso-Marreros IA, Piñarreta-Cornejo PK, Mayta-Tristán P, Bernabé-Ortiz A. Effect of a salt-reduction strategy on blood pressure and acceptability among customers of a food concessionaire in Lima, Peru. Nutr Diet. 2019;76(3):250-6.

20. Beer-Borst S, Hayoz S, Eisenblätter J, Jent S, Siegenthaler S, Strazzullo $P$, et al. RE-AIM evaluation of a one-year trial of a combined educational and environmental workplace intervention to lower salt intake in Switzerland. Prev Med Rep. 2019;16:100982.

21. Dorresteijn J, Graaf Y, Zheng K, Spiering W, Visseren F. The daily 10 kcal expenditure deficit: A before-and-after study on low-cost interventions in the work environment. BMJ open. 2013;3.

22. He FJ, MacGregor GA. Effect of longer-term modest salt reduction on blood pressure. Cochrane Database Syst Rev. 2004(3):Cd004937.

23. MacGregor GA, Markandu ND, Sagnella GA, Singer DR, Cappuccio FP. Doubleblind study of three sodium intakes and long-term effects of sodium restriction in essential hypertension. Lancet. 1989;2(8674):1244-7.

24. Sacks FM, Svetkey LP, Vollmer WM, Appel LJ, Bray GA, Harsha D, et al. Effects on blood pressure of reduced dietary sodium and the Dietary Approaches to Stop Hypertension (DASH) diet. DASH-Sodium Collaborative Research Group. N Engl J Med. 2001;344(1):3-10.

25. Gonçalves C, Pinho O, Padrão P, Santos C, Abreu S, Moreira P. Knowledge and Practices Related to Added Salt in Meals by Food Handlers. Revista Nutrícias. 2014:14-7.

26. Bertino M, Beauchamp GK, Engelman K. Long-term reduction in dietary sodium alters the taste of salt. Am J Clin Nutr. 1982;36(6):1134-44.

27. Blais CA, Pangborn RM, Borhani NO, Ferrell MF, Prineas RJ, Laing B. Effect of dietary sodium restriction on taste responses to sodium chloride: a longitudinal study. Am J Clin Nutr. 1986;44(2):232-43.

28. Kim GH, Lee HM. Frequent consumption of certain fast foods may be associated with an enhanced preference for salt taste. J Hum Nutr Diet. 2009;22(5):475-80. 29. Lucas L, Riddell L, Liem G, Whitelock S, Keast R. The influence of sodium on liking and consumption of salty food. J Food Sci. 2011;76(1):S72-6. 